\title{
Separability and Non-Individuality Is it possible to conciliate (at least a form of) Einstein's realism with quantum mechanics?
}

\author{
Décio Krause* \\ Logic and Foundations Research Group \\ Department of Philosophy \\ Federal University of Santa Catarina \\ dkrause@cfh.ufsc.br
}

(31.08.2005)

\begin{abstract}
In this paper we argue that physical theories, including the most recent ones, even if only implicitly, talk of 'objects' (or 'things') of some sort (really, of several sorts), and question the logico-mathematical apparatus we still use to formulate them, taking into account what such theories presuppose about these entities. I shall point out that despite the discourse (or at least some discourses) goes in the direction of assuming that these quantum objects would be 'new entities' of some kind, distinct from the traditional physical objects of classical physics, the logico-mathematical framework we use is still the old one, grounded on classical logic and set theory, which are committed to atavistic concepts based on individuals and distinguishable things, in complete disagreement with our present day conception of quanta. So, the use of such apparatus would impede us to be in complete agreement with the ontological commitment the theories of quanta seem to propose. Thus, I move in the direction of joining those who try to question the 'logic of quantum mechanics' from a different point of view, looking for a formal rationale for a new ontology.
\end{abstract}

*I would like to thank Newton da Costa, Silvio Chibeni, and Ian Thompson for useful comments and criticism. 
As a consequence of this move, we can revisit Einstein's ideas on physical reality and see that, from the perspective of considering a new kind of object, here termed 'non-individuals', it is possible to sustain that they still obey some of Einstein's conditions for 'physical realities', so that it will be possible to talk of a 'principle of separability' in a sense which is not in complete disagreement with quantum mechanics. So, Einstein's departure from quantum mechanics might be softened at least concerning a form of his realism (locality still remains a challenge of course), for we guess that the incompatibility between quantum mechanics (field theories included) and some form of 'separability' makes sense only if the objects of discourse are thought as 'classical' objects, typical of classical ontology.

Key words: quantum objects, non-individuality, separability, quasi-sets, extensional ontology.

AMS 2000 Subject Classification: Primary: 03E70 Secondary: 81P10 2001 PACS 01.70.+w, 02.10.-v, 03.65.Ta

To be presented at the Simposium "A. Einstein: científico y filósofo. Centenario de una nueva visión del mundo", Cali, Colombia, 26-30 September 2005.

\section{Introduction}

Present day physicists generally work as if they 'merely' should explain why the world is as it is, leaving the issue of discussing what is its real stuff to philosophers. Steven Weinberg, for example, said that "the aim of physics at its most fundamental level is not just to describe the world, but to explain why it is the way it is" [50, p. 175]. So, although in a vague way, we may say that their main activity would be to ask if their formalisms (mathematical schema) give adequate answers to the questions posed on physical systems, without giving importance (as philosophers do) to the real stuff of 'reality' at all. ${ }^{1}$

This way of thinking is consonant with the evolution of the discourse of physicists and (some of) their theories, which moved from a world of objects (for instance, from Galileu's discourse about 'bodies', and of course Newtonian physics)

\footnotetext{
${ }^{1}$ Yet there is a confusion here, for we might say that 'to explain' (or 'to describe') the world is just to indicate what is to be real. Anyway, we do not aim to enter in this philosophical discussion, but only to remark that standard ontological questions do not occupy physicists in general. For instance, no one (I suppose) is asking whether strings or branes posed by present day physics do really exist; they are part of 'the framework', to use Carnap's terminology [6].
} 
to a world of mathematical equations, which sometimes are said to refer only to potentialities, typical of present day quantum mechanics (Bohr's school) [36]. Quantum mechanics, in particular, turned out to be just a mathematical formalism which allows us to compute probabilities related to physical events. According to this school, in short, these values exist only after a measurement being performed; before that, there is a limitation on what can be said about quantum systems, as for instance questions about their individuation. In a certain sense, the objects of physics are ruled out from the theory, which makes reference to them only in an indirect way. ${ }^{2}$ Really, according to Heisenberg, as it is well known, quantum theory is not something which describes nature, but only our knowledge about nature. What I shall sustain is that what has been ruled out from quantum theories is the concept of 'classical object', but that quantum objects are still present at least in the informal semantics of these theories. By the way, in his [19], H. Folse sustained a similar opinion regarding Bohr's view. As Folse said, "I argue that Bohr's viewpoint is more correctly identified as a rejection of only one form of realism [so, he wouldn't be an anti-realist, as it is usually claimed], namely, one which would be compatible with the world-view of classical physics".

This schema is particularly important if we take into account philosophers' interest in 'models' of scientific theories, mainly in consonance with the so-called 'semantic view of theories' [47], for a scientific theory is generally taken to be involved with some interpretation in addition to its mathematical formalism [42]. So, a look for a way of representing its models is in order, that is, we should pay attention on the metamathematical stuff where these models are built. ${ }^{3}$ To give the reader an idea of what I am talking about, let me take a simple example taken from mathematics (the case involving physical theories follows essentially the same idea, although more complicated). Let us suppose group theory, $\mathcal{G}$. A model of $\mathcal{G}$ is a set theoretical structure composed of a non empty set endowed

\footnotetext{
${ }^{2}$ We shall leave the discussion about string-like theories out, like superstring theory for, as said Glashow, it does not exist as a theory yet: "[superstring theory] does not now exist, may never exist, and is probably not even a sensible concept" [25, p. 77]. In a book available at the web, Ian Thompson discussed several 'quantum ontologies', namely, the ontologies of particles, of events, of waves, of propensities and the wave-particle complementarity ontology, all of then with advantages and, of course, great problems. But, as he says, all of them are ways of describing "different possibilities for the individual things which exist in the quantum world" (cf. [48, §4.3].

${ }^{3} \mathrm{~N}$. da Costa has insisted that, for instance, the notion of truth (in the usual Tarskian sense) depends not only on the set theory used in the metamathematics, but also on the particular model of such a theory [9]. Thus, if there are 'objects' involved, our metamathematical resource would be able to describe them as the theory suppose, for instance, as indistinguishable entities. More on this below.
} 
with a binary operation, so that the group axioms are true in this structure in the ordinary (Tarskian) sense. From this point of view, a theory is something like an ordered pair $T=\langle F, M\rangle$, where $F$ is the mathematical counterpart of the theory and $M$ is the class of its models (see [13, Chap. 3], and also [47, Chap. 2]).

Even when speaking of quanta as things not yet actualized, but as 'potentialities', physicists and philosophers still make reference to 'objects' of some sort for, if not, what are to be these potentialities? Something unimaginable? On the other hand, if we regard the 'creation' of the objects to a extreme view which says that before measurement nothing exist at all, of what are our equations, which refer to such potentialities, talking about? In fact, even in quantum field theories, where the idea of particles (thought as 'little objects') is subsumed in a formalism involving 'fields', when something like "Being entangled with" (which has been taken perhaps as the 'basic' property of quantum mechanics) has to be taken as a property that is predicated of at least two quantum systems [18], we may ask "Be entangled with what?". That is, the talk of 'something' remains; due to our atavistic languages and ontology taken from classical physics (and classical logic and mathematics), we could say following Redhead that the 'particle grin' (read: some idea of a thing) is still present in the discourse of quantum theories. We think that we should be able to express the existence of these entities in some way compatible with the theory's presuppositions, for instance, as non-individuals. As we shall see below, by using standard mathematics (and logic) we can do that only by using the resource of ad hoc postulates, like symmetry-like postulates.

Apparently, there is no escape from this kind of talk and the ad hoc resources if we use our objectual standard languages, grounded on classical physics, classical logic and standard mathematics, all of them built on concepts of (apparently) individual and distinguishable things which surround us. ${ }^{4}$ Several authors, from Schrödinger on, have remarked this fact [46]. Recently, some philosophers of physics, and physicists with a philosophical mind, have proposed to update this kind of discourse by admitting that we may be faced with a 'new kind of object', that is, that present day physics face us with a new kind of ontology [32], [3], [49]. Some of them, like Bartels, move to postulate that quanta are not the building blocks of quantum reality, but that these are to be 'Davidsonian events' instead [2]. Shimon Malin also uses a similar analogy, but speaking in terms of 'processes' in Whitehead's sense [36] (see also [?]).

Anyway, philosophical interpretations apart, we should recognize that it is

\footnotetext{
${ }^{4}$ The issue related to the origins of laws of logic and mathematics and their dependence on a 'classical view' of the world is discussed by several authors; for instance, see [8].
} 
quite difficult to think of a discourse which would not be about 'objects' of some kind, even if 'they' are fields, events, or something else. As Toraldo di Francia has insisted, objectuation, that is, the act of dividing up the world in objects to talk of them, is a primary act of our mind, and bases the way we construct our discourse about the world [49].

In this paper I shall not be deeply occupied with the question of the nature of quantum objects. I will just take as a point of departure that the discourse of physics, even those of the most recent theories, are about 'objects' of a sort (perhaps of several sorts), like particles, waves, fields, or strings, and to question the logico-mathematical apparatus we still use to formulate our theories about them. In short, I shall point out that despite the discourse (or at least some discourses) about possibly 'new entities', the logico-mathematical framework we use is still the old one, grounded on classical logic and set theory, which is committed to atavistic concepts based on individuals and distinguishable things, in complete disagreement with our present day conception of quanta. So, I move in the direction of joining those who try to question the 'logic of quantum mechanics' from a different point of view, that is, not in studying abstract algebraic lattices [15], but in looking for a formal rationale for such a new ontology, which I shall mention in some of its main characteristics below.

As a consequence of this move, we can revisit once again Einstein's ideas on physical reality and see that, from the perspective of considering a new kind of object, it is possible to sustain that they obey some of his conditions for 'physical realities', so that it will be possible to talk of a 'principle of separability' in a sense which is not in complete disagreement with quantum mechanics. In short, I sustain that we can speak (in mathematical terms) of 'distinct realities', although non-individual ones. So, Einstein's departure from quantum mechanics might be softened at least concerning a form of his realism (locality still remains a challenge of course), for we guess that the incompatibility between quantum mechanics (field theories included) and some form of 'separability' makes sense if the objects of discourse are thought as 'classical' objects, typical of classical physics (that is, as individuals).

\section{Talking of objects}

Let us take some few examples from present day physics. Consider Bose-Einstein condensates (BECs) first. What are they? In short, and the mathematical formalism just gives to this idea an adequate way to describe these entities, BECs are 
collections of quanta (atoms, ions, etc.) cold near to the absolute zero so that they behave as something macroscopic (I am emphasizing the relevant words which remind us to the 'classical ontology'). But BECs are composed of several quanta which are in the same state, although there are no differences among them. But no physicists, I believe, will say neither that these quanta (which form a BEC), are the very same entity, nor that there is a difference from one each other, or then that we cannot determine the number of quanta in a BEC. In other words, the 'single blob' these quanta form, the 'super atom', is still a group of quanta which have coalesced into the blob. ${ }^{5}$ Finally, we should discard the confusion between what a theory uses in its descriptions and what the theory refers to. That is, in saying for instance that a theory description of an entity is not a description of an individual, but of a type, we are making a confusion between language and reference. We may use types to refer to electrons, say, but it seems beyond doubt that the supposed theory is about electrons.

As a second example, let us take entangled quanta. According to the usual theory (Copenhagen school), quanta become actual only after a measurement. Before measurement, nothing can be said about their individual behaviour; in the popular jargon, they do not exist except as potentialities. Okay, but even if not actualized yet, for example having turned to be light points in a TV screen, what are they? This is a difficult question of course, so is to explain why they make a particular choice of a point when collapsing, that is, when interacting with an atom in the screen. ${ }^{6}$ But note that in these situations we are still making reference to quanta, yet potential ones. The formalism still 'talks' of them when we write the relevant non-factorizable functions and describe entangled systems. To write out these functions, we label them, name them, refer to them, yet we suppose that they are not individuals, in the sense that there is no property that can be possessed by them as individuals. In other words, they are there, existing at least in the informal semantics associated with the relevant functions (or state vectors) -see below.

Peter Mittelstaedt, in a recent paper, has joined M. Redhead and P. Teller (1991) in describing the main features of 'classical' physical objects, so characterizing what he calls classical ontology, $O(C)$ [39], [43]. Let us summarize their claims. The objects of $O(C)$ have (among others) the following characteristics: (1) they are continuants, in the sense that an individual at one time can be identified as the same one that existed at an earlier time -that is, they have genidentity, a term

\footnotetext{
${ }^{5} \mathrm{~A}$ good informal description of these condensates can be seen at the BEC Homepage, http: //www . colorado. edu/physics/2000/bec/.

${ }^{6}$ Shimon Malin has developed Dirac's view that "Nature makes a choice (...) when there is no longer a possibility of interference" [36, p. 127].
} 
coined by Reichenbach; (2) they are objects of predication, and may bear properties; (3) they are individualisable, in the sense that there is 'something' (Locke: "I don't know what" [34, Chap. XXIII, Book II]) which confers then an identity; (4) they have identity; (5) they can be counted, named, tagged, labeled.

From the logical point of view, we can say that for every property $P$, we have that $P$ or its counter property, to use Mittelstaedt words, holds [39]. If we understand this 'counter property' as the negation of $P$, namely, $\neg P$, then a form of the excluded middle principle is in order: $P \vee \neg P$. Furthermore, it seems clear that we shouldn't accept that both contradictory properties hold at once $;^{7}$ so, the contradiction rule $\neg(P \wedge \neg P)$ seems to be also valid. In addition, for every property $P$, we still have what Mittelstaedt has called the 'complete determination' of an object (attributed to Kant): "if all possible predicates are taken together with their contradictory opposites then one each pair of contradictory opposites must belong to it" (hence, we could add, each property belongs to it). In other words, there is valid here a rule called in some contexts 'the explosion rule', or Duns Scotus Rule: from a contradiction every proposition can be deduced, for $P \wedge \neg P \rightarrow Q$ is to be true. If we pursue these analogies, it is clear that we shall obtain the rules of classical logic. Really, we can say that classical logic was built with $O(C)$ in our minds.

A problem raises when we consider quantifiers. 'Classical' quantifiers, due to their semantic interpretation, presuppose identity [12]; when we say that "There exists an object so and so", and this is true, we mean that there exists a set of objects from which we are talking about and that there is an element of this set so and so. Furthermore, this object can be always distinguished from any other: it is an individual. A similar idea holds for the universal quantifier. Classical semantics presuppose classical set theory. But sets, according to standard mathematics, are collections of distinguishable objects, which is in complete agreement with $O(C)$. So, it is not easy to explain in precise terms what a physicist intends to say when she speaks that "there exists an electron so and so", in the case that these entities are taken to be indiscernible from other electrons. But we shall leave this point as a problem to be further investigated, perhaps by adapting plural quantifiers to the quantum real. ${ }^{8}$

\footnotetext{
${ }^{7}$ The reader may be thinking in complementary propositions here. But we would like to say that we do not regard complementary propositions as being the negation of one another, strictly speaking. In another work ([11]), we have approached the idea, which will not be considered here, mainly because complementarity does not appear in classical physics.

${ }^{8}$ Plural quantifiers, introduced by G. Boolos, formalize things like "There are apples on the table" (or perhaps better in our case, "There are electrons so and so") without -apparently- any
} 
All of this suggest to the reader that this kind of discourse will be not suitable concerning quantum objects. As Schrödinger suggested, "[w]e have taken over from previous theory [classical mechanics and $O(C)$ ] the idea of a particle and all the technical language concerning it. This idea is inadequate. It constantly drives our mind to ask for information which has obviously no significance. Its imaginative structure exhibits features which are alien to the real particle" [46, p. 202]. It is beyond doubt that by 'real particle' he is referring to quantum objects. As for an example of an information 'without significance' we recall Schrödinger's own account on identity as applied to quanta.

In a series of public lectures given by Schrödinger at the Institute for Advanced Studies in Dublin in 1950, subsequently published as Science and Humanism [45], he writes that, in the face of quantum physics,

"... we have ... been compelled to dismiss the idea that ... a particle is an individual entity which retains its 'sameness' forever. Quite the contrary, we are now obliged to assert that the ultimate constituents of matter have no 'sameness' at all. (...) I beg to emphasize this and I beg you to believe it: It is not a question of our being able to ascertain the identity in some instances and not being able to do so in others. It is beyond doubt that the question of 'sameness', of identity, really and truly has no meaning" [45, pp. 17]

The above lines suggest to the reader some of the main traits of quantum objects, which we shall summarise as a form of 'quantum ontology', $O(Q)$ (see also Mittelsteadt op.cit.): non-individuality, lack of identity, indistinguishability, absence of genidentity (identity trough time, or re-identification), etc. This move has a consequence, recalled by G. Toraldo di Francia:

"[h]istorians have perhaps been blinded by the glamor of relativity and quantum mechanics and are likely to describe these theories as the revolutions of the early 20th century. Yet, I believe that a much more important development took place at the turn of the century, a development that future historians will probably place in more adequate perspective. This is the discovery of nomological objects (...) Nomological objects are described by physical law; or, perhaps each class of them represents a physical law." [49]

commitment to sets; [33]. 
Classical objects, according to him, are not 'nomological', since "... their individual configuration had nothing to do with laws" [49, p. 62] The idea appears to be that in classical mechanics the value of the mass of a particle, say, is not given by law, so that it can have a contingent range of values. As he says, the value of mass is not determined a priori, for a physical law like Newton's second law $F=m . a$ holds for any value of $m$ whatsoever. For a nomological object, on the other hand, this value, and also those of other properties such as charge, angular momentum etc., is well determined and 'prescribed' by physical law. For instance, one can formulate the law that a mass $m=9.1 \times 10^{-23} \mathrm{~g}$ must always be accompanied by an electric charge $e= \pm 4.8 \times 10^{-10}$ e.s.u., by a spin $\hbar / 2$, and so on, and anything which possesses this set of properties has to be an electron.

As we have already remarked (see [22, Chap. 5]), the crucial 'discovery' he refers to in the above quotation appears to be that of discreteness, in the sense that the value of a particle's charge can be expressed as an integral multiple of the charge of an electron, angular momentum is quantised as multiples of $\hbar / 2$ and so on. In this sense, the values of a property cannot take any of a continuous range of values, which seems to reintroduce a fundamental form of atomism in physics, in terms of which one might assert that the notion of physical object has been 'recovered'. 9

What Toraldo di Francia seems to suggest is that the 'new' object is submerged under certain kind in modern physics, in the sense that an electron, for example, is defined to be that kind of thing which has a mass of $9,1 \times 10^{-23} \mathrm{~g}$, a charge of $4,8 \times$ $10^{-10}$ e.s.u. and so on. The discreteness of these values implies that such kinds characterising electrons, muons, quarks, etc.- are sharply delineated. Thus, the view of objects as 'nomological' ultimately reduces to a form of bundle theory, as Toraldo di Francia himself acknowledges: "In some way, physical objects are today knots of properties, prescribed by physical laws" [49, p. 63].

The 'recovery' of integral numbers inherent in the above discreteness appears to reduce measurement to counting, at least in what respect some quantities like electric charge. But the counting of elementary particles is problematic and here Toraldo di Francia raises concerns about individuality:

"Can we distinguish this and the other, in a system of two electrons? As is well known, this cannot be done: identical particles are indis-

\footnotetext{
${ }^{9}$ However, it is still not clear in what sense these properties are 'well determined' and the objects themselves 'prescribed' by law. After all, the charge of an electron, Planck's constant etc., all represent fundamental constants whose values cannot be obtained by physical law, but we shall not advance this point here; see [22].
} 
tinguishable. Here, cardinal numbers seem to take over the role we had previously attributed to ordinal numbers. A system of identical particles has a cardinality; but we cannot tell which is the first, the second, and so on." (ibid.)

Although there seems to be here a conflation of individuality with distinguishability, a point we can leave aside here (but see [22]), the conclusion that an assembly of quantum particles may have cardinality but not ordinality has an impact on the foundations of logic:

"Think, for instance, that in any formalised theory, we must start by defining a universe of discourse, that is a set of objects we want to talk about. For this to make sense, it must be well determined whether an object belongs or not to the set (extension of the set); moreover, the objects must be distinguishable from one another, so we can tell which is which. These requirements can perhaps be met by mathematical objects (although some scholars strongly oppose the idea). But what happens in the case when our universe of discourse is made up of physical objects? How can we tell that it is determined whether a given electron belongs or not to our system, and how can we tell which electron we are talking about?" [49, p. 65].

The alternatives Toraldo di Francia proposes are to employ fuzzy set theory or to develop an intensional semantics appropriate for such objects. Really, he developed (with M. L. Dalla Chiara) a quaset theory to cope with these questions, which we shall turn to consider later.

All these considerations suggest that $O(Q)$ seems to demand a different logicomathematical framework for describing quantum objects. In this stuff, if we are to talk of 'objects' of some kind, identity and indistinguishability should not be equi-extensional, as they are in classical logic and mathematics -see below-; in particular, collections of quanta should not be regarded as sets (collections of individuals), and so on. Let us reinforce these points in the next section.

\section{More reasons}

We can find other arguments for the need of considering an alternative logicomathematical apparatus to deal with these philosophical issues involving quanta. ${ }^{10}$

\footnotetext{
${ }^{10}$ Let us insist in the philosophical aspect of this need. There are interesting approaches to quantum mechanics given entirely within classical logic and mathematics; see [35]. In these
} 
Suppose that we have a language $\mathcal{L}$ suitable to talk of quantum objects, involving logical and mathematical notation and concepts, and that we intend to define the corresponding semantic concepts as we usually do. ${ }^{11}$ What kind of problem will we be faced with? Such an analysis was done by M. L. Dalla Chiara and G. Toraldo di Francia some time ago [14], ${ }^{12}$ and the points they have emphasised are of course of fundamental importance for whatever discussion on structure and semantics of quantum theories.

Suppose that $\mathcal{L}$ is at least of first order and let $\mathfrak{A}$ be an structure where $\mathcal{L}$ is interpreted. Since this structure is a set-theoretical construct (lets us suppose that we have Zermelo-Fraenkel set theory (ZF) as our metalanguage), $\mathfrak{A}$ involves a domain (set) $D$ of individuals and an interpretation (or denotation) function $\rho$ which assigns appropriate meaning to the non-logical constants of $\mathcal{L} .^{13}$ Of course we can think for generality of $\mathfrak{A}$ as determining a set of possible worlds for each particular physical situation under analysis, so that in this case we would have a set of domains $D_{i}$ and a collection of corresponding interpretation functions $\rho_{i}$. The particular case above happens when the set of possible worlds is a singleton. Then, as recalled by Dalla Chiara and Toraldo di Francia, the following situations can be considered in 'standard' semantics:

(i) Any property $P$ of $\mathcal{L}$ (an unary predicate) is related to a subset $P^{*} \subseteq D_{i}$, while $n$-ary predicates are related to subsets of $D_{i}^{n}$ as usual.

(ii) For each individual $d^{*} \in D_{i}$, the language can be extended to a language containing a name $d$ and an extended interpretation function $\rho^{\prime}$ such that $\rho^{\prime}(d)=$ $d^{*}$.

(iii) If $\mathcal{L}$ is at least of second order, then Leibniz' Principle of the Identitity of Indiscernibles (PII) holds (individuals can be distinguished by at least one property):

$$
\forall x \forall y(x \neq y \rightarrow \exists F(F(x) \wedge \neg F(y)) .
$$

schema, physics of course works, but the philosophical problems we are pointing out have no clear explanation.

${ }^{11}$ Just to emphasise the difficulties present also here, it is useful to recall that Yuri Manin has suggested that quantum mechanics has not its 'own' language, making use of a fragment of standard functional analysis to formulate its concepts [38, p. 84].

${ }^{12}$ See [22] for further references and comments.

${ }^{13}$ In the particular case of physical theories, due to the needs of a higher-order logic, it is reasonable to suppose that the domain $D$ sum up a certain mathematical construction in terms of other sets. In this case, we should talk of a 'species of structures' in the sense of Bourbaki, but we are obviously making things easy, since these details do not concern us here. 
(iv) If $\mathfrak{A}$ refers to a set of possible worlds (physical situations), as in the usual Kripke semantics, we can suppose the existence of a number of world-relations; a particular one may corresponds to a time-order relation as follows: $i<j$ iff the situation $i$ temporally precedes the situation $j$. Of special interest is the relation $\approx$, termed the trans-world identity relation, defined on $U=\cup_{i} D_{i}$ satisfying the following conditions:

(a) For any $d^{*} \in D_{i}$ and any $D_{j}$ there exists at most one $\widehat{d^{*}} \in D_{j}$ such that $d^{*} \approx \widehat{d^{*}}$.

(b) If $d$ is an individual constant of $\mathcal{L}$ (a name) which names individuals in two distinct worlds, say $d^{*} \in D_{i}$ and $\widehat{d^{*}} \in D_{j}$, then $d^{*} \approx \widehat{d^{*}}$. In particular, if $i<j$, the trans-world identity relation between $D_{i}$ and $D_{j}$ is usually called genidentity relation.

In what concerns quantum physics, the interesting remark is that all these standard set-theoretical semantic situations are violated. Dalla Chiara and Toraldo di Francia provided a detailed analysis of the motives why such semantics fails, here only sketched. The first problem is that we should be able to construct a suitable language containing monadic predicates for expressing 'meaningful properties' of the physical systems (in particular, of elementary particles), so as to consider names $d, d^{\prime}, \ldots$ which should be associated to these physical systems at different times. Since indistinguishable quanta cannot be named, for in general we cannot distinguish a physical system (elementary particle) from another, our language cannot be extended with 'names' as indicated at item (ii) above and so we cannot define a suitable interpretation function $\rho$ that univocally determines an element of $D$. As remarked by these mentioned authors, "the problem is not "whether or not we are allowed to introduce names $a_{1}, \ldots, a_{n}$ for the $n$ subsystems (...)' but rather "whether or not we are able to introduce a reasonable denotation function $\rho$ for such names' ".

Furthermore, a physical system can be regarded as represented by a pure state $\psi$, as usual, and time evolution of the system is governed by the Schrödinger equation. The problem is that we cannot say that $\psi$ determines a set of $n$ elements in the standard set theoretical sense, which contradicts condition (i) above. To see why, let us introduce the concept of vagueness in this context. ${ }^{14}$ Philosophers usually say that vagueness is a feature of our languages, and not of the world. For instance, the predicate 'intelligent' is vague, for we might be in doubt either John is or not intelligent, although John is a well defined physical object (a man), an

\footnotetext{
${ }^{14}$ This point was not considered by the mentioned authors, but of course can be related to their ideas.
} 
individual we know very well. That is, John is to be regarded as 'sharp object' (or as an object of $O(C)$ ), while the predicate 'intelligent' is what is vague.

It has been proposed that a suitable semantics for such vague predicates should be developed not within standard set theories, but that we should use fuzzy set theory instead. The reason is that within the standard framework all predicates are 'sharp' in the sense that they are associated to a subset of the domain, called its extension (as indicated at (i) above) so that whatever element of this domain belongs or not to the extension of the predicate, which means that any individual should be classified as intelligent or as not intelligent (the tertium non datur applies here); recall our characterization of $O(C)$ above. It is easy to see why fuzzy sets provide a more adequate semantics, for in using fuzzy sets, we can express in a certain way our ignorance about John's precise location in the rank of intelligent men (see Figure 1). I will not explain the details here (but see [29]), but let me remark that fuzzy sets can be useful just for expressing the situation when a well defined individual (like our friend John) has or not a certain (vague) property -such a semantics express a certain (for the lack of a better term) 'epistemological ignorance' only.

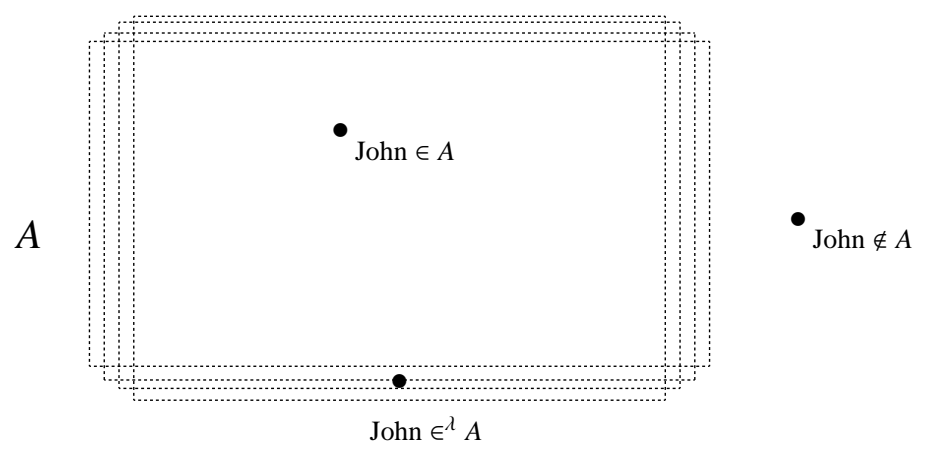

Figure 1: The fuzzy set $A$ of intelligent men. There are three cases to consider: (1) the individual John does belong to the fuzzy set $A$; (2) John does not belong to $A$, and (3) John $\in^{\lambda} A, 0<\lambda<1$. A fuzzy set is still a collection (yet with borderlines not well defined) of individuals.

But in quantum physics, there are certain 'predicates' which are sharp in the sense that physicist know quite well the conditions an individual should obey to have the property ascribed by the predicate, for example the spin. That is, "To have spin up in a certain direction, say $x$-direction", is a sharp condition. But here, since we are within $O(Q)$, there are 'vague objects' instead (like 'events'), 
which induce the consideration of a kind of 'ontological ignorance' in this realm. ${ }^{15}$ This shows that the relationship between the predicates themselves (which stand for the intensions of certain concepts) and their corresponding extensions (the set of the individuals which have the property ascribed by the predicate) becomes distinct from standard semantics. In such cases, it seems clear that we should ask for a semantics which deals with 'imprecise' or 'vague' objects, and indistinguishable objects are good candidates for that, since they would have not a criterion of identity.

Since the extension of a predicate like "To have spin up in the $x$-direction", as applied to a collection of quanta has no well defined extension (for we cannot specify it precisely, except concerning its cardinal, that is, the quantity of quanta having the considered property), ${ }^{16}$ condition (i) above of standard semantics is violated. In other word, collections of quanta should not be regarded as 'sets' (as in standard set theories) but collections of indistinguishable objects should be considered instead. Important to recall that this remark was made also by Yu. I. Manin in the first problem of Problems of Present Day Mathematics, presented at the 1974 meeting to evaluate and to explore the consequences of the famous list of 23 Problems of Mathematics proposed by Hilbert in 1900 [4, p. 76].

To look at (iii), let us first emphasize that classical logic and mathematics (ZF, say) are Leibnizian in a sense: they encompass a theory of identity according to which there cannot be indistinguishable but not identical objects. But it has been shown by several authors that there are strong reasons to suppose that PII is violated in the quantum domain (and there are of course 'set-theoretical' versions of this principle), for we may consider (absolutely) indistinguishable quanta (having all their quantum parameters in common) which of course are not the very same object ([22]). ${ }^{17}$ But here again the problem regarding the quantifiers appear; when we formulate PII as above, by supposing that they apply to quanta, are the used quantifiers 'classical'? We have already made reference to the fact that standard quantifiers presuppose identity. As we see, the formal discussion is not simple indeed.

We shall leave the discussion of item (iv), for we would need much space

\footnotetext{
${ }^{15}$ This idea was developed in a series of previous papers; see [21].

${ }^{16}$ If relativistic concepts are to be considered, then even the cardinal of such a collection is to be questioned; see [49].

${ }^{17}$ Of course we should be careful here in discussing spatial localization, for this would entail a detailed account on the distinction between bosons and fermions due to Pauli'sn principle. But we will not consider this point here, for I guess that the careful reader can understand perfectly well what I intend to say.
} 
for the details; so, we shall just say that Dalla Chiara and Toraldo di Francia have shown that in this 'land of anonymity', as they refer to the quantum world, a world where there are no proper names, the standard forms of Kripke semantics fail, for there are no trans-world identity and the notion of rigid designator cannot be applied [14], among other interesting 'deviations' from classical situations [22].

All of this suggest that we should ask for a mathematical theory encompassing 'collections' which could stand for 'sets' of indistinguishable objects, where no names can be used, no individuation of these objects can be given, but even so they should be considered in aggregates, perhaps having a cardinal number, although not an associated ordinal. This is what quasi-set theory provides. The applications of this theory to the above situations have been suggested in some of the papers listed in the references, but there is still much to be done in this direction.

\section{Is there a place for (some form of) realism?}

It is usual to regard Bohr-Einstein debate (which lived for circa 50 years) as the most fundamental scientific philosophical debate of the XXth century. After Bell's theorem from the sixties, the core of the problem left to be just an epistemological one, concerning if quantum mechanics is a complete theory, and gained the status of what A. Shimony has termed an 'experimental metaphysics' [24, p. 126] (but see also pp. 226 and 243). That is, the guess that there would be non-local phenomena could be tested experimentally! We shall not revise this discussion here, but just recall that, as it is commonly understood, the violation of Bell's inequalities provides the grounds for the claim that Bohr was the winner ${ }^{18}$ and Einstein's local-realism should be ruled out [36], [16]. Local realism, let us recall, combines locality, that is, the hypothesis that nothing (no body, no information) can travel faster than light and realism (a name for a lot of conceptions) which can be summed up by saying that an outside world exists independently of our will or of our consciousness.

Einstein had strong reasons to believe in locality (see [27], [7]), and he regarded science as concerning the 'real' due to his commitment to a principle of separability (PS), which seems to be the core of his philosophical doctrine [26, 27], [40]. ${ }^{19}$ As put by Howard [26], after the EPR paper Einstein presented his own version of the incompleteness argument (it is today well known that Ein-

\footnotetext{
${ }^{18}$ This interpretation can be discussed; see [7].

${ }^{19}$ Howard calls our attention to a letter from Einstein to Eduard Study in which he says that "I concede that the natural sciences concern the 'real', but I am still not a realist" [27, §5].
} 
stein did not agreed with Podolski's final version of the paper), which is entailed by the conjunction of two assumptions: locality and separability. By separability, we mean (following Howard) that spatially separated systems have associated with them independent real states of affairs [27]. To Einstein, suggests Howard, realism "... is not a philosophical doctrine about the interpretation of scientific theories or the semantics of theoretical terms ... [but it] is a physical postulate, one of the most interesting kind" (ibid.), which bases his conception of physical reality.

To understand the link between realism and separability, let us quote Einstein, according to whom physical reality entails

"... that what we conceive as existing ('actual') should somehow be localized in time and space. That is, the real in one part of space, A, should (in theory) somehow 'exist' independently of that which is thought as real in another part of space, B. If a physical system stretches over the parts $\mathrm{A}$ and $\mathrm{B}$, then what is present in $\mathrm{B}$ should somehow have an existence independent of what is present in $\mathrm{A}$. What is actually present in B should thus not depend upon the type of measurement carried out in the part of the space, A; it should also be independent of whether or not, after all, a measurement is made in A." (apud [27])

That is, as put by Howard, "[r]ealism is thus the thesis of spatial separability, the claim that spatial separation is a sufficient condition for the individuation of physical systems, and its assumption is here made into almost a necessary condition for the possibility of an intelligible science of physics" (ibid.). Of course, in a letter to Born, Einstein has also said that

"... if one abandons the assumption that what exists in different parts of space has its own, independent, real existence, then I simply cannot see what it is that physics is meant to describe. For what is thought to be a 'system' is, after all, just a convention, and I cannot see how one could divide the world objectively in such a way that one could make statements about parts of it". (cf. [22, chap. 4])

The criticism usually addressed to this position seems to be centered in the fact (for instance, given by Howard himself) that separability acts as a principle of individuation. As individuals, quantum objects would not be able to enter in 
entangled states, for in this case they should encompass peculiar properties, contrary to the idea of the entanglement. But I would like to remark that this is true only if they are to be taken as individuals according to $O(C)$. If this is so, then of course separability would be a sufficient condition for individuality and then the violation of Bell inequalities would entail, first, that we must give up separability for quantum particles in entangled states and hence, secondly, that when in such states these particles cannot be regarded as individuals. In other words, Bell's Theorem implies non-individuality [22, Chap. 4].

But this is not necessarily so. The discourse about 'separated' things could be read in terms of the affirmative that, in some sense, we may admit the existence of collections of quanta with cardinal greater than one, that is, of more than one quanta of a certain kind (for instance, in supposing a BEC), although these quanta could have not individuality. That is, these quanta, although forming a collection, would be not individuals at all or, at least, not individuals in the sense of classical ontology. The difficulty in articulating such a case would be a source for the problems involving quantum ontology. Of course, we can 'suppose' such a view, for in principle it is easy to imagine that there can be absolutely indistinguishable things. But how to provide a right mathematical description of these 'objects' (for the lack of an adequate word)?

The standard solutions, as it is well known, make use of symmetry postulates. Let us revise this point a little bit in order to emphasise our ideas. Let us consider the distribution of two indistinguishable (physicists say 'identical') quanta, initially termed \#1 and \#2, distributed over two states $\mathbf{A}$ and $\mathbf{B}$. Let us further suppose that each resulting arrangement is accorded equal probability. As it is well known, these assumptions conduce to four possibilities, given by adequate vectors in a suitable Hilbert space:
(1) $\left|\psi_{\mathbf{A}}^{1}\right\rangle \otimes\left|\psi_{\mathbf{B}}^{1}\right\rangle$
(2) $\left|\psi_{\mathbf{A}}^{2}\right\rangle \otimes\left|\psi_{\mathbf{B}}^{2}\right\rangle$
(3) $\left|\psi_{\mathbf{A}}^{1}\right\rangle \otimes\left|\psi_{\mathbf{B}}^{2}\right\rangle$
(4) $\left|\psi_{\mathbf{A}}^{2}\right\rangle \otimes\left|\psi_{\mathbf{B}}^{1}\right\rangle$.

In this Dirac's 'bra' and 'ket' notation, the superscripts (labels) 1 and 2 mean that they can be distinguished, since different states are characterized by different state functions.

In classical statistical mechanics (Maxwell-Boltzmann), (3) and (4) are counted as distinct and given equal weight in the assignment of probabilities. This informally means that the situation where we have one object in each state is given a weight of two, corresponding to the two arrangements or complexions that may 
be formed by a permutation of the objects. The difference in the states implies that the objects are to be regarded as individuals in some way, that is, the difference of the states (3) and (4) should be attributed to something 'transcending' the objects' properties, sometimes spelled out in terms of some underlying 'haecceity' or 'primitive thisness' or, more typically, the spatio-temporal location of the objects [41] (see [22]).

In the quantum case the situation is distinct. We shall restrict our attention to the two standard forms of statistics, Bose-Einstein (B-E) and Fermi-Dirac (F-D). Here, while (1) and (2) remain the same, (3) and (4) must be substituted by (3') and (4'):

$$
\left(3^{\prime}\right) \frac{1}{\sqrt{2}}\left(\left|\psi_{\mathbf{A}}^{1}\right\rangle \otimes\left|\psi_{\mathbf{B}}^{2}\right\rangle+\left|\psi_{\mathbf{A}}^{2}\right\rangle \otimes\left|\psi_{\mathbf{B}}^{1}\right\rangle\right) \quad\left(4^{\prime}\right) \frac{1}{\sqrt{2}}\left(\left|\psi_{\mathbf{A}}^{1}\right\rangle \otimes\left|\psi_{\mathbf{B}}^{2}\right\rangle-\left|\psi_{\mathbf{A}}^{2}\right\rangle \otimes\left|\psi_{\mathbf{B}}^{1}\right\rangle\right) .
$$

Here, (1), (2) and (3') give B-E statistics, while (4') gives F-D. This is standardly taken to reflect the fact that arrangements obtained by a permutation of the objects do not feature in the relevant counting in quantum statistics.

More formally, it should be noted that states of quantum systems (single or many-object systems) are represented by unitary vectors $\Psi$ in a Hilbert space $\mathcal{H}$. For many object systems the Hilbert space is constructed by forming the tensor product of the component objects' Hilbert spaces. For a system consisting of two indistinguishable objects, the Hilbert space is $\mathcal{H}_{\text {two }}=\mathcal{H}_{1} \otimes \mathcal{H}_{2}$, where the subscripts ' 1 ' and ' 2 ' label the objects, and $\mathcal{H}_{1}=\mathcal{H}_{2}=\mathcal{H}$ for they are indistinguishable. If the objects are in the pure states $\phi$ and $\psi$ respectively, then the composite system is in the (pure) state $\Psi=\phi \otimes \psi$. The observables $\hat{O}$ of a quantum system are represented by Hermitian operators acting upon that system's Hilbert space. A permutation of the objects over states is represented by an operator and these 'permutation operators' form a group known ever since Hermann Weyl as the Permutation Group [5]. These permutation operators are projections (hence have eigenvalues \pm 1$)$ and act upon $\Psi$ as follows: (1) $\hat{P}_{i d}(\Psi)=(\phi \otimes \psi)$ and (2) $\hat{P}_{\phi \psi}(\Psi)=(\psi \otimes \phi)$.

The Hamiltonian operator, $\hat{H}_{\Psi}=\hat{H}(\phi \otimes \psi)$, of the composite system is symmetric with respect to $\phi$ and $\psi$. Hence, $\hat{H}_{\Psi}$ is invariant under the action of the permutation group of permutations of the composite objects' labels, that is, $[\hat{H}, \hat{P}]=0$, for any $\hat{P}$. The 'fact' that object permutations are not counted is understood in terms of there being no measurement that we could perform which would result in a discernible difference between permuted (final) and unpermuted (initial) states. This is represented in the formalism by insisting that every physical observable $\hat{O}$ commutes with every permutation operator $\hat{P}$, that is, $[\hat{O}, \hat{P}]=0, \forall \hat{O}, \forall \hat{P}$. Expressing 
this formally, we have the so-called 'Indistinguishability Postulate' (IP), that is, for any arbitrary state $\psi$, Hermitian operator $\hat{O}$, and permutation operator $\hat{P}$,

$$
\langle\psi|\hat{O}| \psi\rangle=\langle\hat{P} \psi|\hat{O}| \hat{P} \psi\rangle=\left\langle\psi\left|\hat{P}^{-1} \hat{O} \hat{P}\right| \psi\right\rangle .
$$

Since (IP) allows for the possibility of forms of quantum statistics which are different from the 'standard' Bose-Einstein and Fermi-Dirac kind, if one wants to restrict the formalism to the latter kinds only, then a further condition, known as the 'Symmetrization Postulate' (SP) must be applied (this corresponds to a 'symmetry condition') [23]. This dictates that states of indistinguishable object systems must be either symmetrical or anti-symmetrical under the action of the permutation operators (corresponding to the Bose-Einstein and Fermi-Dirac cases respectively). The difference between (SP) and (IP) can be expressed as follows: (SP) expresses a restriction on the states for all observables, $\hat{O}$; whereas (IP) expresses a restriction on the observables, $\hat{O}$, for all states.

Now, (IP) seems to run counter to the point of regarding the objects as individuals and labeling them; from the point of view of the statistics, the object labels are otiose. The implication, then, is that the objects can no longer be considered to be individuals, that is, they are, in some sense, 'non-individuals'. This conclusion expresses an idea that came from Schrödinger, Born, Heisenberg, Weyl, Hesse and Post at least (see [22] for historical details): classical objects are individuals but quantum objects are not. Post, for instance, drew on the distinction between form and substance, arguing that what quantum statistics indicates is the ontological primacy of the former over the latter [41].

As suggested above, our aim is to say that entangled systems, BECs and so on, might be viewed as collections of non-individuals with cardinal greater than one. If this is assumed, then we may regard the elements of such collections as 'distinct realities', although they cannot be individualized by a property whatsoever. Distinct spatio-temporal location here is then just a name for "cardinal grater than one', and so a kind of 'separability' is in order, once the idea of a collection with cardinal greater than one induces (following $O(C)$ ) the idea of more than one object, hence of 'distinct physical realities' in a sense. This way, such an hypothesis can be considered, and it entails neither individuality nor incompatibility with quantum mechanics. Of course, one could say, this way of thinking may work at the informal level, but the problem is how to express that in an adequate formalism. As shown above, the standard ways of dealing with the problem is by means of ad hoc alternatives: we label the quanta first, writing \#1 and \#2, say, and then use some symmetry condition to get at the situation where permutations 
of indiscernible quanta are not regarded to be observable, for instance in taking vectors like (3') above. But, as emphasised by Post and Manin at least, indiscernibility should be attributed right at the start [41] (see [22]). So, we should look for a mathematical formalism which express this fact directly; this is what quasi-set theory aims to do.

We have presented this theory in other works (see [28], [22]), so that we shall recall here only its basic traits, mainly concerning its underlying logic, never explicated in details before.

\section{Quasi-set theory: logical aspects}

Let $\mathcal{L}$ be a first-order language whose primitive logical symbols are $\neg, \vee$ and $\forall(\wedge, \rightarrow, \leftrightarrow$ and $\exists$ are defined as usual). We still admit auxiliary symbols like parentheses and the comma, so as two unary predicates $m$ and $M$, two binary predicates $\equiv$ and $\in$ and an unary functional symbol $q c$. The individual variables are $x_{1}, x_{2}, \ldots$, but we shall use $x, y, \ldots$ to denote them. We assume a set of individual constants, denoted by $a, b, c, \ldots$ which act as contants for a certain type, or kind, that is, they do not have the role of names of object of the domain, for we are supposing (in our informal semantics) that these objects may be non-individuals (perhaps we could call them type-individuals). So, when using a constant, say $c$, this does not mean that we are referring to an specific object (named $c$ ), but to an object of a kind (or type). This is more or less what happens in physics, when in speaking of an electron, for instance in saying that an electron was released from a certain atom during ionization, physicists in general are not naming a particular electron by the simple fact that whatever name they use gives the electron only a 'mock-individuality' [14, p. 266]. Terms and formulas of $\mathcal{L}$ are defined as usual. When $x \in y$, we say that $x$ is an element of $y$. The term $q c(x)$ is called 'the quasicardinal' of $x$. If $m(x)$, we say that $x$ is an $m$-atom, while $M(x)$ says that $x$ is an $M$-atom. These last ones will have all the properties ascribed to individuals (Urelemente) by classical logic and mathematics.

Let us define quasi-sets and the extensional equality as follows:

$$
\begin{aligned}
& \text { (1) } Q(x) \stackrel{\text { def }}{=} \neg m(x) \wedge \neg M(x) \\
& \text { (2) } x=_{E} y \stackrel{\text { def }}{=}(Q(x) \wedge Q(y) \wedge \forall z(z \in x \leftrightarrow z \in y)) \vee(M(x) \wedge M(y) \wedge \forall z(x \in \\
& z \leftrightarrow y \in z)))
\end{aligned}
$$


Thus, a quasi-set is something which is not an atom, while extensionally identical objects are either quasi-sets having the same elements or $M$-atoms which belong to the same quasi-sets. So, extensional equality does not apply to $m$-atoms. From now on, when we say 'identical', we mean 'extensionally identical'. Since I have made a critical reference to the use of quantifiers in their usual sense in this realm, it would be interesting, I suppose, to make clear what kind of suppositions we are making when speak of 'Exists a certain object so and so'. Thus, let us see the postulates of the underlying first-order logic of quasi-set theory $\mathfrak{Q}$, whose language is $\mathcal{L}$. They are the following ones, where $\alpha, \beta$ and $\gamma$ denote formulas:

$$
\begin{aligned}
& \text { (A1) } \alpha \vee \alpha \rightarrow \alpha \\
& \text { (A2) } \alpha \rightarrow \alpha \vee \beta \\
& \text { (A3) } \alpha \vee \beta \rightarrow \beta \vee \alpha \\
& \text { (A4) }(\alpha \rightarrow \beta) \rightarrow((\gamma \vee \alpha) \rightarrow(\gamma \vee \beta)) \\
& \text { (MP) } \alpha, \alpha \rightarrow \beta / \beta \\
& \text { (A5) } \forall x \alpha(x) \rightarrow \alpha(c) \text {, where } c \text { is a type-constant. } \\
& \text { (A6) } \beta \rightarrow \alpha(x) / \beta \rightarrow \forall x \alpha(x) \text {, provided that } x \text { is not free in } \beta \text {. } \\
& \text { (इ } \left.{ }_{1}\right) \forall x(x \equiv x) \\
& \left(\equiv_{2}\right) \forall x \forall y(x \equiv y \rightarrow y \equiv x) \\
& \left(\equiv_{3}\right) \forall x \forall y \forall z(x \equiv y \wedge y \equiv z \rightarrow x \equiv z) \\
& \left(\equiv_{4}\right) \forall x \forall y\left(x={ }_{E} y \rightarrow(\alpha(x) \rightarrow \alpha(y)) \text {, where } \alpha(x)\right. \text { is a formula whatever } \\
& \text { and } \alpha(y) \text { is obtained by replacing } x \text { by } y \text { at one or more free occurrences } \\
& \text { of } x \text { in } \alpha(x) \text {. }
\end{aligned}
$$

In words, the axioms involving quantification show the admissible way of using the quantifiers; when we write $\exists x \alpha(x)$, where $\alpha$ is a formula where $x$ appears free, this intuitively means that there exists an object which obeys one of the primitive predicates of the language so that $\alpha$ holds. Since we are not assuming that the semantics of our logic is to be formulated in a standard set theory like ZF, we are not necessarily committed with individuals here (elements of some set).

That is, our variables range over collections (formally: quasi-sets) whose elements may be indistinguishable (non-individuals). So, let us insist once more, 
when we say, for instance that from $\exists x \alpha(x)$ we deduce $\alpha(c)$ for some $c$, this $c$ should not be read as an individual constant (that is, as a name of an object of the domain), but as a generalized constant, a type-constant as explained above, which denote an individual of a certain class (say, an electron, but not a particular electron). Thus, the quantifiers have here a wide informal interpretation, coping also with with non-individuals.

Axiom $\left(\equiv_{4}\right)$ entails that the substitutivity principle does not hold for $m$-atoms. Here the reader may be worried, for it seems that indistinguishable atoms should be substituted one another in whatever situation. But in assuming that, we would introduce difficulties in the theory, for then $\equiv$ would be standard identity, and nothing new would be achieved. Anyway, a more interesting result, to be presented below, plays the role of such a principle for $m$-atoms (see theorem 1). Axioms $\left(\equiv_{1}\right)-\left(\equiv_{3}\right)$ say that $\equiv$, which we call the relation of indistinguishability, is reflexive, symmetric and transitive. This will be important in taking a quasi-set whose only elements are $m$-atoms and passing the quotient by the relation $\equiv$, for obtaining collections (quasi-sets) of indistinguishable objects. Hence there is a sense in speaking that some of them may be 'of the same species' (partake the relation $\equiv$ ), as indicated above.

The other postulates are ZFU-like axioms (Zermelo-Fraenkel with Urelemente) and provide the grounds for the construction of a hierarchy of quasi-sets, similar to the cumulative hierarchy in standard set theory (see Figure 1). Some specific axioms which should be mentioned for our needs here are: (a) the atoms are empty, in symbols, $\forall x \forall y(x \in y \rightarrow Q(y))$; (b) no atom is both an $m$-atom and an $M$-atom. We shall not repeat the other postulates here (see [22], [30]), but we shall only informally comment on some results that follow from them.

Intuitively speaking, a quasi-set is a collection of objects, so that some of them may be indistinguishable (yet not identical). This of course is not a strict 'definition' of a quasi-set, giving no more than an intuitive account of the concept. It is also easy to prove that the extensional identity has all the properties of standard identity (in particular, one of the postulates say that if neither $x$ nor $y$ are $m$-atoms, then $x \equiv y \rightarrow x={ }_{E} y$ ).

The theory enable us to consider that (extensional) identity and indistinguishability are separated concepts; that is, these concepts do not reduce to one another as in standard set theories. So, in this theory, contrary to standard mathematics (say, that one obtained from ZFU -Zermelo-Fraenkel with Urelemente), we may have indistinguishable but not identical objects.

It is possible to define a translation from the language of ZFU into the language of $\mathfrak{Q}$ in such a way so that there is a 'copy' of ZFU in $\mathfrak{Q}$ (this is the 'classical' part 


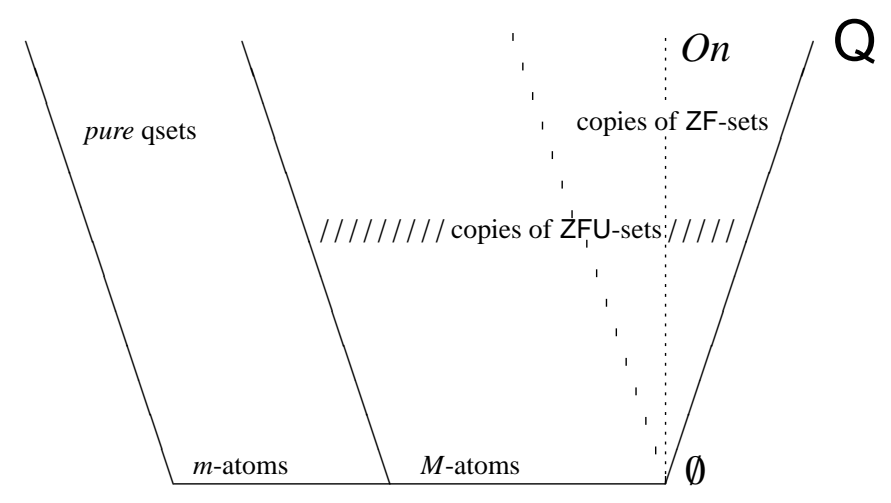

Figure 2: The Quasi-Set Universe. On stands for the class of the ordinals.

of $\mathfrak{Q}$ ). In this part (see the Figure 1 again), all the usual mathematical concepts can be defined, and the 'sets' (better, the ' $Q$-sets') turn out to be those quasisets whose transitive closure (this concept is like the usual one) does not contain $m$-atoms.

In $\mathfrak{Q}$ there may exist quasi-sets whose elements are $m$-atoms only, called 'pure' quasi-sets whose elements may be indistinguishable (in the sense of partaking the primitive indistinguishability relation $\equiv$ ) and the axioms provide the grounds for saying that nothing in the theory can distinguish the elements of such an $x$ from one another. The primitive concept of quasi-cardinal, given by adequate postulates, provides the intuitive idea of the 'quantity' of objects in a quasi-set. Since it is axiomatical, the concept of quasi-cardinal is independent of a concept of ordinal. Within the theory, the idea that there is more than one entity in $x$ is expressed by an axiom which states that the quasi-cardinal of the power quasi-set of $x$ (the concept of subquasi-set is like that of standard set theory) has quasicardinal $2^{q c(x)}$, where $q c(x)$ is the quasi-cardinal of $x$ (which is a cardinal obtained in the 'copy' of ZFU just mentioned). Now, what exactly this supposition means?

Consider the three protons and the four neutrons in the nucleus of a ${ }^{7} \mathrm{Li}$ atom. As far as quantum mechanics goes, nothing distinguishes neither the three protons nor the four neutrons from one each other. If we regard the three protons as forming a quasi-set, its quasi-cardinal should to be 3 , and there is no apparent contradiction in saying that there are also 3 subquasi-sets with 2 elements each, despite we can't distinguish them or their elements, and so on. The same happens 
with quantum entangled systems before measurement. So, it is reasonable to postulate that the quasi-cardinal of the power quasi-set of $x$ is $2^{q c(x)}$. Whether we can distinguish among these subquasi-sets is a matter which does not concern logic.

In other words, we may consistently (with the axiomatics of $\mathfrak{Q}$, supposing them consistent) reason as if there are three entities in our quasi-set $x$, but $x$ must be regarded as a collection for which it is not possible to discern its elements as individuals. The theory does not enable us to form the corresponding singletons. The grounds for such kind of reasoning has been delineated by Dalla Chiara and Toraldo di Francia as partly theoretical and partly experimental. Speaking of electrons instead of protons, they note that in the case of the helium atom we can say that there are two electrons because, theoretically, the appropriate wave function depends on six coordinates and thus "... we can therefore say that the wave function has the same degrees of freedom as a system of two classical objects" $\left[14\right.$, p. 268]. ${ }^{20}$ Dalla Chiara and Toraldo di Francia have also noted that, "[e]xperimentally, we can ionize the atom (by bombardment or other means) and extract two separate electrons ..." (ibid.).

Of course, the electrons can be counted as two only at the moment of measurement; as soon as they interact with other electrons (in the measurement apparatus, for example) they enter into entangled states once more. It is on this basis that one can assert that there are two electrons in the helium atom or six in the $2 p$ level of the sodium atom or (by similar considerations) three protons in the nucleus of a ${ }^{7} \mathrm{Li}$ atom (and it may be contended that the 'theoretical' ground for reasoning in this way also depends on these experimental considerations, together with the legacy of classical ontology). On this basis it is stated the axiom of 'weak extensionality' of $\mathfrak{Q}$, which says that those quasi-sets that have the same quantity of elements of the same sort (in the sense that they belong to the same equivalence class of indistinguishable objects) are indistinguishable.

This axiom has interesting consequences. There is no space here for the details, but let us mention that in standard (extensional) set theories, if $w \in x$, then of course $(x-\{w\}) \cup\{z\}=x$ if and only if $z=w$. That is, we can 'exchange' two elements without modifying the original arrangement if and only if these elements are the same elements, by force of the axiom of extensionality (that is, nothing was done at all). But in $\mathfrak{Q}$ we can prove the theorem below, where $z^{\prime}$ (and similarly $w^{\prime}$ ) stand for a quasi-set with quasi-cardinal 1 whose only element is indistinguish-

\footnotetext{
${ }^{20}$ This is associated to what Schrödinger had anticipated, in saying that this kind of formulation "gets off on the wrong foot" by initially assigning object labels and then permuting them before extracting combinations of appropriate symmetry [46].
} 
able from $z$ (respectively, from $w$-the reader shouldn't think that this element is identical to either $z$ or $w$, for the relation of equality doesn't apply here; the other set theoretical operations can be understood according to their usual definitions):

Theorem 1 [Unobservability of Permutations] Let $x$ be a finite quasi-set (its quasi-cardinal is a natural number, obtained in the 'classical' part of the theory) such that $x$ does not contains all the objects indistinguishable from $z$, where $z$ is an $m$-atom such that $z \in x$. If $w \equiv z$ and $w \notin x$, then there exists $w^{\prime}$ such that

$$
\left(x-z^{\prime}\right) \cup w^{\prime} \equiv x
$$

Proof: (For details, see [30]). If $t \in z^{\prime}$ does not belong to $x$, then $x-z^{\prime}={ }_{E} x$ and so we may admit the existence of $w^{\prime}$ such that its unique element $s$ belongs to $x$ (for instance, $z$ itself); then $\left(x-z^{\prime}\right) \cup w^{\prime}=_{E} x$. If $t \in z^{\prime}$ belongs to $x$, then $q c\left(x-z^{\prime}\right)={ }_{E} q c(x)-1$ for we can prove in $\mathfrak{Q}$ that, for all qsets $x$ and $y$, if $y \subseteq x$ and $x$ is finite -that is, its quasi-cardinal is a natural number- (which we are of course supposing in this case), then $q c(x-y)=_{E} q c(x)-q c(y)$. Thus, take $w^{\prime}$ such that its element is $w$ itself (which can be supposed for, although we cannot prove this within the theory, it can be supposed), and so it results that $\left(x-z^{\prime}\right) \cap w^{\prime}={ }_{E} \emptyset$. Hence, since $\forall_{Q} x\left(q c(\mathcal{P}(x))={ }_{E} 2^{q c(x)}\right.$ ) (here we are using relativized quantifiers in the usual sense) in $\mathfrak{Q}$, we have that $q c\left(\left(x-z^{\prime}\right) \cup w^{\prime}\right)={ }_{E} q c(x)$. This intuitively says that both $\left(x-z^{\prime}\right) \cup w^{\prime}$ and $x$ have the same quantity of indistinguishable elements. So, by applying the weak extensionality axiom, we have the theorem.I

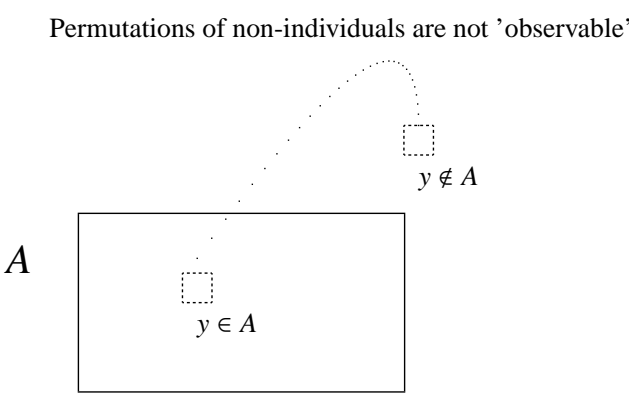

Figure 3: Quasi-sets: Either the non-individual $y$ belongs to the quasi-set $A$ or it does not. Here, $y$ does not act as a name for an individual. Furthermore, permutations of indistinguishable $m$-atoms generate a quasi-set indistinguishable from the original one.

Supposing that $x$ has $n$ elements, then if we 'exchange' their elements $z$ by correspondent indistinguishable elements $w$ (set theoretically, this means performing 
the operation $\left.\left(x-z^{\prime}\right) \cup w^{\prime}\right)$, then the resulting quasi-set remains indistinguishable from the original one by force of our 'weak' axiom of extensionality (which uses $\equiv$ instead of identity). In a certain sense, it is not important whether we are dealing with $x$ or with $\left(x-z^{\prime}\right) \cup w^{\prime}$. So, within $\mathfrak{Q}$ we can express that 'permutations are not observable', without necessarily introducing ad hoc postulates like IP (equation (4)) above.

The postulates enables us to see a difference between quasi-sets and fuzzy sets. In fuzzy set theory, as it is well-known, the counter-domains of the characteristic functions are not $\{0,1\}$, but the whole interval $[0,1]$. So, within fuzzy set theory, the 'ignorance' (or vagueness) is with respect to the place an individual has in a fuzzy set (see again Figure 2 above and 3 below), while in quasi-set theory, vagueness concern the non-individuals themselves. ${ }^{21}$

\section{Conclusion}

Quasi-set theory has other applications, for instance in deriving quantum statistics without the needs of postulating certain symmetry conditions. In short, by using quasi-relations, we can express a particular distribution of $v_{i}$ particles in $k_{i}$ states in a way that the counting of the resulting states agrees with the quantum statistics, without the need of assuming that permutations are not regarded as observable. In other words, quantum statistics (for instance Bose-Einstein and Fermi-Dirac) result 'natural' within the quasi-set framework. For details on this point, see [31], [30].

To sum up, even if with the resources of our physical theories (that is, in posing what Carnap calls 'internal questions' [6]), we do not describe individuals, but only types, we cannot forget the objectivity of these theories, for these types refer to 'objects' of a kind, perhaps non-individuals. Thus, we could say, following Auyang, that "... people reject quantum objects because they are different but all their argument shows is that there is nothing like classical objects in the quantum realm, not that there is no quantum object" $[1$, p. 5]. So, an adequate mathematical language would be considered for expressing the main semantic properties involving quanta, a language which would be 'natural' and free from the necessities of ad hoc postulates like symmetry conditions.

Thus, if we are able to talk (in mathematical terms) of collections of entities which are of course not 'the same ones' but so that there are no (in principle)

\footnotetext{
${ }^{21}$ This point regarding 'vague objects' has been discussed elsewhere; see [20], [21].
} 
differences among them, we guess that Einstein's realism, at least if read as assuming the needs for a certain objectivity in taking of 'objects' of some sort, does not need to be completely ruled out from quantum theories.

\section{References}

[1] Auyang, S.Y., (1995), How is Quantum Field Theory Possible?, New York, Oxford Un. Press.

[2] Bartels, A., (1999), 'Objects or events? Towards an ontology for Quantum Field Theory', Phil. Sci. - Procceedings 66, pp. S170-S184.

[3] Bitbol, M., (1996), Schrödinger's Philosophy of Quantum Mechanics, Kluwer Ac. Press (Boston Studies in the Philosophy of Science, Vol. 188).

[4] Browder, F.E. (ed.), (1976), Mathematical Problems Arising from Hilbert Problems, Proceedings of Symposia in Pure Mathematics, Vol. XXVIII, Providence, American Mathematical Society.

[5] Bueno, O. and French, S., (1999), 'Infestation or pest control: the introduction of group theory into quantum mechanics', Manuscrito 22 (2), pp. 37-68.

[6] Carnap, R., (1947), 'Empiricism, semantics, and ontology', The Journal of Philosophy, 20-40.

[7] Chibeni, S.S., (1997), Aspects of the physical description of reality (in Portuguese), Centro de Lógica, Epistemologia e História da Ciência, Universidade Estadual de Campinas, Coleção CLE n. 21.

[8] da Costa, N.C.A., (1980), Ensaio Sobre os Fundamentos da Lógica, São Paulo, Hucitec/EdUSP (2nd. ed. Hucitec 1994).

[9] da Costa, N.C.A., (2005), 'On Tarski's definition of truth', preprint.

[10] da Costa, N.C.A. and Krause, D., (1994), 'Schrödinger logics', Studia Logica 53, pp. 533-550.

[11] da Costa, N.C.A. and Krause, D., (2004), 'Remarks on the applications of paraconsistent logic to physics', at philsci-archive.pitt.edu/archive/00001566.

[12] da Costa, N.C.A., French, S. and Krause, D., (2005), 'Identity and individuality: logical aspects', in preparation. 
[13] Dalla Chiara, M.L. and Toraldo di Francia, G., (1981), Le Teorie Fisiche: un'analisi formale, Boringhieri.

[14] Dalla Chiara, M.L. and Toraldo di Francia, G., (1993), 'Individuals, kinds and names in physics', in Corsi, G. et al. (eds.), Bridging the gap: philosophy, mathematics, physics, Dordrecht, Kluwer Ac. Press, pp. 261-283.

[15] Dalla Chiara M.L., Giuntini, R. and Greechie, R., (2004), Reasoning in quantum theory: sharp and unsharp quantum logics, Kluwer Ac. Pu.

[16] d'Espagnat, B., (1979), 'The quantum theory and reality', Scientific American 241 (5), pp. 158-181.

[17] d'Espagnat, B., (1983), In search of reality, Springer-Verlag.

[18] Esfeld, M., (2004), 'Quantum entanglement and a metaphysics of relations', forthcoming in St. Hist. Phil. Modern Physics.

[19] Folse, Jr., H.J., (1986), 'Complementarity and scientific realism', in P. Weingartner and G. Dorn (eds.), Foundations of Physics, A selection of papers contributed to the Physics Section of the 7th International Congress of Logic, Methodology and Philosophy of Science (Vienna, Hölder-Pichler-Yempsky), pp. 93-101.

[20] French, S. and Krause, D., (1995), 'Vague identity and quantum non-individuality', Analysis 55 (1), pp. 20-26.

[21] French, S. and Krause, D., (2003), 'Quantum Vagueness', Erkenntnis 59, pp. 97124.

[22] French, S. and Krause, D., (2005), Identity in Physics: a Historical, Philosophical and Formal Analysis, forthcoming.

[23] French, S. and Rickles, D.P., (2002), 'Understanding permutational symmetry', forthcoming.

[24] Ghirardi, G., (2005), Sneaking a look at God's cards: Unraveling the Mysteries of Quantum Mechanics, Princeton Un. Press.

[25] Glashow, S.L., (1999), 'Does quantum field theory need a foundation?', in Cao, T. Y. (ed.), Conceptual Foundations of Quantum Field Theory, Cambridge, Cambridge University Press, pp. 74-88.

[26] Howard, D.A., (1985), 'Einstein on locality and separalility', Studies in History and Philosophy of Science 16, pp. 171-201. 
[27] Howard, D.A., (2004), 'Einstein's Philosophy of Science', The Stanford Encyclopedia of Philosophy (Spring 2004 Edition), Edward N. Zalta (ed.), at plato.stanford.edu/archives/spr2004/entries/einstein -philscience.

[28] Krause, D., (1992), 'On a quasi-set theory', Notre Dame Journal of Formal Logic 33, pp. 402-411.

[29] Krause, D., (2002), 'Why quasi-sets?', Bol. Soc. Paran. Mat. 20 (1/2), pp. 73-92.

[30] Krause, D., (2003), 'The mathematics of non-individuality', Instituto de Estudos Avançados, Universidade de São Paulo, Série Lógica e Teoria da Ciência n.46, Março 2003.

[31] Krause, D., Sant'Anna,A.S. and Volkov, A. G., (1999), 'Quasi-set theory for bosons and fermions: quantum distributions', Foundations of Physics Letters 12 (1), pp. 51-66.

[32] Lévy-Leblond, J.-M., (2002), 'On the nature of quantons', Science and Education.

[33] Linnebo, ., (2004), 'Plural Quantification', The Stanford Encyclopedia of Philosophy (Winter 2004 Edition), Edward N. Zalta (ed.), at plato.stanford.edu/archives/win2004/entries/plural -quant.

[34] Locke, J., (1994), An Essay Concerning Human Understanding, at www . arts. cuhk. edu.hk/Philosophy/Locke/echu/

[35] Ludwig, G., (1983), Foundations of quantum mechanics, I, Springer-Verlag.

[36] Malin, S., (2001), Nature loves do Hide: Quantum Physics and the Nature of Reality, a Western Perspective, Oxford U. Press.

[37] Manin, Yu.I., (1976), 'Mathematical Problems I: Foundations', in Browder, F. E. (ed.): 1976, Mathematical Problems Arising from Hilbert Problems, Proceedings of Symposia in Pure Mathematics, Vol. XXVIII, Providence, American Mathematical Society, p. 36.

[38] Manin, Yu.I., (1977), A course in mathematical logic, Springer-Verlag.

[39] Mittelstaedt, P., (2003), 'Quantum physics and classical physics - in the light of quantum logic', at arXiv:quant-ph/0211021 v2 3 Apr 2003.

[40] Paty, M., (2005), Personal communication by e-mail.

[41] Post, H. (1963), 'Individuality and physics', The Listener 70, pp. 534-537; reprinted in Vedanta for East and West 32, pp. 14-22. 
[42] Post, H. (1971), 'The incompleteness of quantum mechanics or the Emperor's missing clothes', in Bastin, T., ed. (1971), Quantum Theory and Beyond: Essays and Discussions arising from a Colloquium, Cambridge Un. Press, pp. 275-282.

[43] Redhead, M. and Teller, P., (1991), 'Particles, particle labels, and quanta: the toll of unacknowledged metaphysics', Foundations of Physics 21, pp. 43-62.

[44] Rhorlich, R., (1999), 'On the ontology of QFT', in Cao, T. Y. (ed.), (1999), Conceptual Foundations of Quantum Field Theory, Cambridge, Cambridge University Press, pp. 357-367.

[45] Schrödinger, E., (1951), Science and Humanism, Cambridge, Cambridge Un. Press.

[46] Schrödinger, E., (1998), 'What is an elementary particle?', reprinted in Castellani, E. (ed.), Interpreting bodies: classical and quantum objects in modern physics, Princeton Un. Press, pp. 197-210.

[47] Suppes, P., (2002), Representation and Invariance os Scientific Structures, CSLI Pu., Stanford.

[48] Thompson, I.J., (2003), Philosophy of Nature and Quantum Reality, www.generativescience.org/books/pnb.

[49] Toraldo di Francia, G. (1978), 'What is a physical object?', Scientia 113, pp. 57-65.

[50] Weinberg, S., (1993), Dreams of a Final Theory: the Search for the Fundamental Laws of Nature, Vintage. 